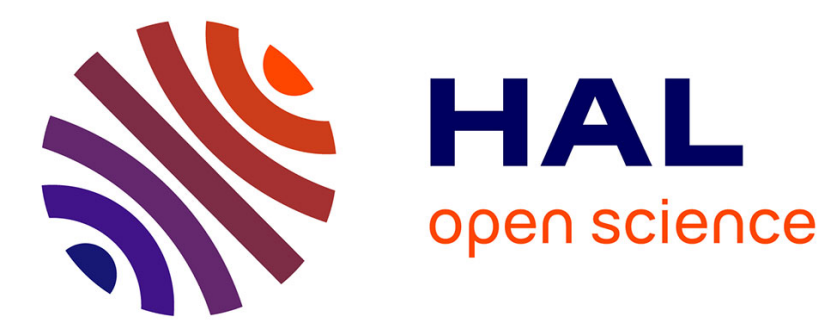

\title{
Statistical properties of genealogical trees
}

\author{
Bernard Derrida, Susanna C. Manrubia, Damian Zanette
}

\section{To cite this version:}

Bernard Derrida, Susanna C. Manrubia, Damian Zanette. Statistical properties of genealogical trees. Physical Review Letters, 1999, 82 (9), pp.1987-1990. 10.1103/PhysRevLett.82.1987 . hal-03285611

\section{HAL Id: hal-03285611 https://hal.science/hal-03285611}

Submitted on 21 Jul 2021

HAL is a multi-disciplinary open access archive for the deposit and dissemination of scientific research documents, whether they are published or not. The documents may come from teaching and research institutions in France or abroad, or from public or private research centers.
L'archive ouverte pluridisciplinaire HAL, est destinée au dépôt et à la diffusion de documents scientifiques de niveau recherche, publiés ou non, émanant des établissements d'enseignement et de recherche français ou étrangers, des laboratoires publics ou privés. 


\title{
Statistical Properties of Genealogical Trees
}

\author{
Bernard Derrida, ${ }^{1}$ Susanna C. Manrubia, ${ }^{2}$ and Damián H. Zanette ${ }^{3}$ \\ ${ }^{1}$ Laboratoire de Physique Statistique de l'École Normale Supérieure, 24 rue Lhomond, F-75231 Paris 05 Cedex, France \\ ${ }^{2}$ Fritz-Haber-Institut der Max-Planck-Gesellschaft, Faradayweg 4-6, 14195 Berlin, Germany \\ ${ }^{3}$ Consejo Nacional de Investigaciones Científicas y Técnicas, Centro Atómico Bariloche e Instituto Balseiro, \\ 8400 S.C. de Bariloche, Río Negro, Argentina
}

(Received 14 October 1998)

We analyze the statistical properties of genealogical trees in a neutral model of a closed population with sexual reproduction and nonoverlapping generations. By reconstructing the genealogy of an individual from the population evolution, we measure the distribution of ancestors appearing more than once in a given tree. After a transient time, the probability of repetition follows, up to a rescaling, a stationary distribution which we calculate both numerically and analytically. This distribution exhibits a universal shape with a nontrivial power law which can be understood by an exact, though simple, renormalization calculation. Some real data on human genealogy illustrate the problem, which is relevant to the study of the real degree of diversity in closed interbreeding communities. [S0031-9007(99)08610-X]

PACS numbers: 87.10. $+\mathrm{e}, 05.20 .-\mathrm{y}, 05.40 .-\mathrm{a}, 64.60 . \mathrm{Ak}$

Modern man appeared on Earth some $10^{5}$ years ago $[1,2]$. At that time, a few social groups totaling several thousands of individuals were occupying small settlements, most probably in Africa [3]. Nowadays, we are faced with about $5 \times 10^{9}$ human beings on Earth, whose lineages could be, in principle, traced back to that time. Each human being has two parents, four grandparents, and, in general, $2^{n_{g}}$ ancestors in the $n_{g}$ th upper generation. Going backwards in time until the first group of anatomically modern Homo sapiens - some 4000 generations ago-we should find $2^{4000} \sim 10^{1200}$ ancestors in each genealogical tree. However, the total human population at those early times was probably only a few thousands. The answer to this apparent paradox is simple: A given individual appears more than once in a genealogical tree [4], even in very distant branches, indicating that many of the ancestors were in fact close relatives. A repeated individual generates a whole repeated branch in the tree, and the further we move into the past, the more frequent the repetitions will be. This is the result of mating inside a finite population, the size of which sets an upper bound to the maximal number of ancestors for a given individual.

These repetitions are particularly apparent when we are faced with a small closed interbreeding population. Royal genealogy provides us with a nice example, since nobles usually married within their own castes. As an illustration to the problem, we have analyzed the repetitions in the genealogical tree of the English king Edward III (13121377) [5]. It contains almost $10^{3}$ individuals, some of which appear more than once (and up to six times) in his tree. We show in Fig. 1 the function $F(r)$, defined as the quotient between the number $M(r)$ of ancestors which appear $r$ times in the tree and the total number of different ancestors $N_{t}: F(r)=M(r) / N_{t}$.

We study here the statistics of repetitions in genealogical trees as a function of the population size and the gen- eration in the past that we are looking at. The question that we are addressing can be put in the more general context of genetic diversity [6,7]. In fact, an important factor in the variability of natural populations is the diversity displayed, in the genealogical history of every individual, by his ancestors themselves and by their weights in the present genome. Here we calculate these weights in a simple neutral model, with no selection, no change in the population size, and no geographical isolation. Possible effects of these on genealogies and genetic diversities are discussed in $[4,8,9]$.

We have started by performing numerical simulations of a simple neutral model of a closed population evolving under sexual reproduction with nonoverlapping generations.

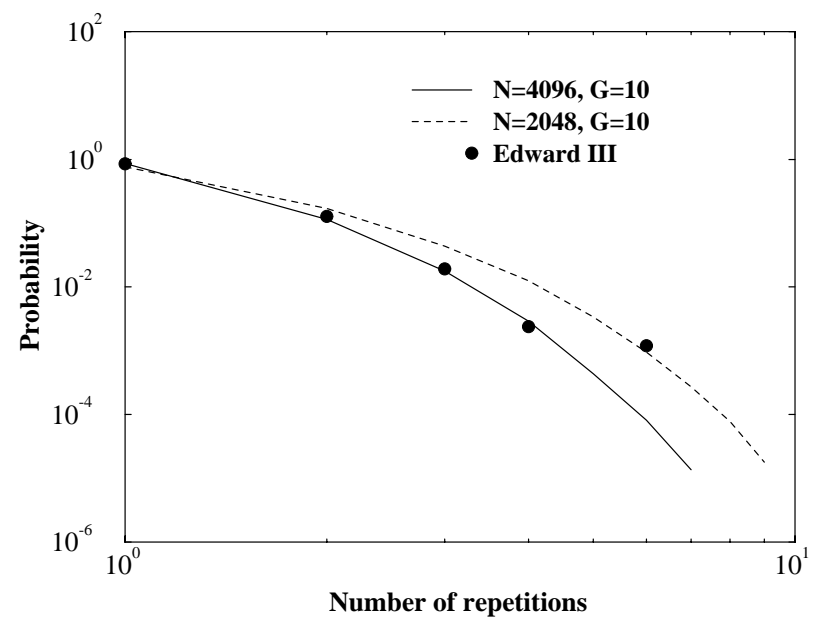

FIG. 1. Probability of ancestor repetitions in the genealogical tree of the king Edward III [5]. The continuous and dashed lines represent the results of simulations of $F(r)$ in a closed population with $2^{11}$ and $2^{12}$ individuals for our model. Averages have been performed over the ten first generations of $10^{3}$ independent trees. 
In our model the population size is fixed to be $N$ for all generations. The population is equally divided into two groups, representing males and females. At every generation, we form heterosexual pairs at random and assign them a certain number of descendants according to a Poisson distribution. This is done by choosing for each male or each female a pair of parents at random in the previous generation [10]. After a number $G$ of generations, the tree of each of the individuals in the youngest generation is reconstructed.

We have first calculated the distribution $F(r)$ of repetitions in this model for a population of $N=2^{11}$ and $N=2^{12}$ individuals. This might be a rough estimate of the number of noble people at the time of Edward III. After $G=10$ generations, we compute the probability of repetitions in the whole tree (notice that in the real world generations often overlap and thus the same person might be found in different generations; this possibility is absent in our model). The result of our simulations is compared with the real data displayed in Fig. 1. We observe an acceptable agreement, although we should say that the distribution $F(r)$ depends rather strongly on $G$ and $N$ and that the agreement is often worse for other reasonable choices of these two parameters.

We have also measured the probability of repetitions $H\left(r, n_{g}\right)$ at every past generation $n_{g}=1, \ldots, G$, that is, the probability that any individual at generation $n_{g}$ in the past appears $r$ times in the tree of an individual at generation $0\left[n_{g}=1\right.$ corresponds to the parents, $n_{g}=2$ to the grandparents, and so on; note that $H\left(0, n_{g}\right)$ is simply the probability that an individual is not present in a tree after $n_{g}$ generations]. In the first few generations (parents, grandparents, etc.), if the population size $N$ is large, the probability of finding an individual more than once in a tree is very small. As a consequence $H\left(r, n_{g}\right)$ decreases with $r$ when $n_{g}$ is small. Going further in the past, at some point two "brothers" will appear in the tree of an individual, and from then on these two branches will coincide. From then on, more and more repetitions will occur.

The distribution $H\left(r, n_{g}\right)$ is shown in Fig. 2. It changes its shape during a transient period of the order of $\log N$ generations. [Note that an important difference between $F(r)$ shown in Fig. 1 and $H\left(r, n_{g}\right)$ is that for $F$ we counted only those individuals present in one particular genealogical tree whereas for $H$ we count the whole population at generation $n_{g}$ in the past.] Clearly, we have $\sum_{r \geq 0} H\left(r, n_{g}\right)=1$ and $\sum_{r} r H\left(r, n_{g}\right)=2^{n_{g}}$. In Fig. 2 we see for $N=2^{15}$ the function $H\left(r, n_{g}\right)$ for different generations before and after reaching the stationary shape. For $n_{g}$ small, $H\left(r, n_{g}\right)$ decreases with $r$, meaning that repetitions are very unprobable. As $n_{g}$ increases, the number of repetitions increases and $H\left(r, n_{g}\right)$ exhibits a maximum and a shape which becomes stationary.

If we rescale the distribution $H\left(r, n_{g}\right)$ by plotting as in Fig. 3 the distribution

$$
P(w) \equiv 2^{n_{g}} H\left(r, n_{g}\right) / N,
$$

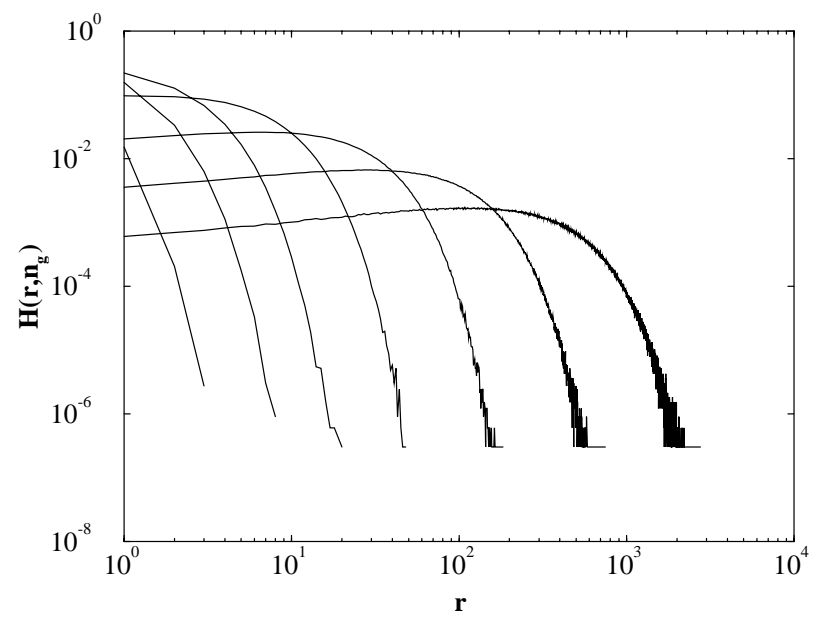

FIG. 2. Distribution $H\left(r, n_{g}\right)$ of $r$ repetitions after $n_{g}$ generations $\left[H\left(0, n_{g}\right)\right.$ is not shown]. The distribution changes after roughly $\log N$ generations from a decreasing function of $r$ to a distribution with a maximum. The generations shown are $n_{g}=9,13,15,17,19,21$, and 23 for a population with $N=2^{15}$. We have averaged over 100 independent runs.

versus the weight

$$
w \equiv r N / 2^{n_{g}},
$$

all the distributions of Fig. 2 (after a transient period) collapse on a single stationary function. Figure 3 represents the function $P(w)$ for several values of $n_{g}$ after the transient period obtained for a population of $N=$ $2^{20}$ individuals. We observe that the left tail of $P(w)$ is a power law, $P(w) \sim w^{\beta}$, and a least squares fit to our numerical results in the domain $w \in\left(10^{-4}, 10^{-1}\right)$ returns $\beta \simeq 0.302$. In addition to the exponent $\beta$, one can accurately measure the moments of $\left\langle w^{n}\right\rangle=\int w^{n} P(w) d w$ of $P(w)$ as well as the fraction $S\left(n_{g}\right)$ of the total population

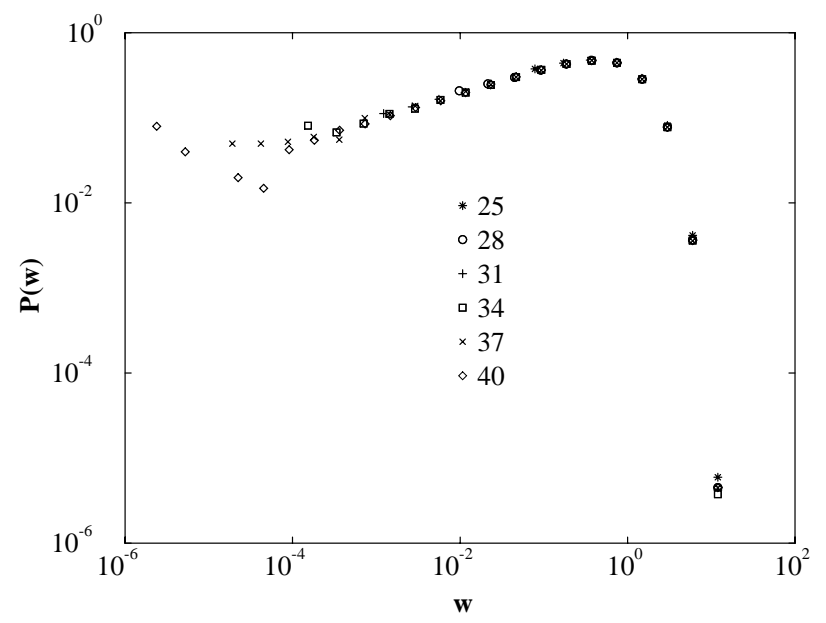

FIG. 3. Data collapse for the rescaled distribution of repetitions $P(w)$ after the transient period. Averages have been performed over $10^{3}$ independent trees for a population size $N=2^{20}$. 


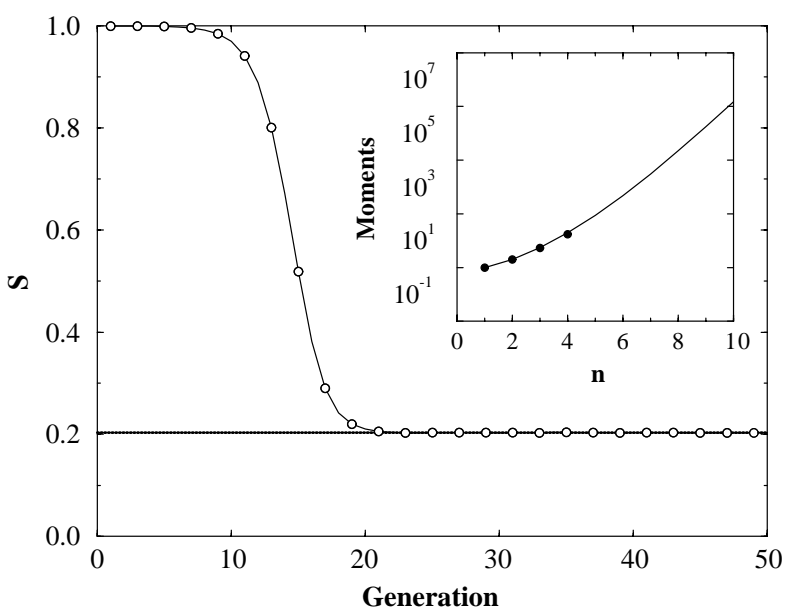

FIG. 4. Dependence of $S\left(n_{g}\right)$ on the generation $n_{g}$ for a population with $N=2^{15}$. The numerical asymptotic value is $S\left(n_{g} \rightarrow \infty\right) \simeq 0.2031$. The bold dotted line is the predicted theoretical value $S=g(-\infty)=0.20318787 \ldots$ In the inset, we represent the first ten moments $\left\langle w^{n}\right\rangle$ for the distribution $P(w)$. The continuous line corresponds to numerical results, while solid circles stand for the theoretical predictions.

in the oldest generation which is absent from a given genealogical tree. Figure 4 contains our numerical estimates for $S\left(n_{g}\right)$. Figure 4 also shows the first moments of the distribution $P(w)$. As can be seen, even when the number of potential ancestors in the tree is much larger than the number of individuals in the population, not all of those give contributions to the present. In fact, the proportion of individuals without descendants reaches a fixed value, $S\left(n_{g} \rightarrow \infty\right) \simeq 0.2031$.

The distribution $P(w)$ can be understood analytically by the following argument: If we consider the genealogical tree of an individual, say individual $i=1$ at the 0th generation, the weights $w$ of his ancestors can be traced back according to the following algorithm. From (1) and (2) we have $w_{i}(0)=N$ for $i=1$ and $w_{i}(0)=0$ for $i \neq 1$. Then the weights of the ancestors at generation $n_{g}+1$ in the past, with $0 \leq n_{g} \leq G-1$, are given by

$$
w_{i}\left(n_{g}+1\right)=\sum_{j \text { children of } i} \frac{w_{j}\left(n_{g}\right)}{2} .
$$

When $N$ is large, the probability $p_{k}$ that an individual at generation $n_{g}+1$ in the past has $k$ children at generation $n_{g}$ becomes a Poisson distribution

$$
p_{k}=2^{k} e^{-2} / k ! \text {. }
$$

Now if for large $N$ we consider that the weights of the children of any given individual are uncorrelated (this can be viewed as an approximation, but in fact, by calculating pair correlations between the weights in our model, one can show that for large enough $N$ this approximation becomes exact), we obtain from (3) and (4) that any weight at generation $n_{g}+1$ is the sum of $k$ independent identically distributed weights at generation $n_{g}$ with $k$ itself randomly chosen according to (4). Then if $g_{n_{g}}(\lambda)$ is the generating function of the weights at generation $n_{g}$ in the past,

$$
g_{n_{g}}(\lambda)=\left\langle e^{\lambda w_{i}\left(n_{g}\right)}\right\rangle,
$$

it follows from (3) [and the fact that for large $N$ the $w_{j}\left(n_{g}\right)$ are uncorrelated] that it satisfies

$g_{n_{g}+1}(\lambda)=\sum_{k=0}^{\infty} \frac{2^{k} e^{-2}}{k !}\left[g_{n_{g}}(\lambda / 2)\right]^{k}=e^{-2+2 g_{n_{g}}(\lambda / 2)}$.

This recursion has the form of a renormalization group transformation. Together with the initial condition

$$
g_{0}(\lambda)=1+\left(e^{\lambda N}-1\right) / N,
$$

it determines all the generating functions $g_{n_{g}}(\lambda)$. When $n_{g} \rightarrow \infty$, the generating function converges to a limit $g(\lambda)$ solution of

$$
g(\lambda)=e^{2 g(\lambda / 2)-2} .
$$

All the information on the shape of the stationary solution $P(w)$ is contained in the solution of (7). For example, one can expand the $g(\lambda)$ solution of (7) in a power series and find that

$$
\begin{aligned}
g(\lambda)= & 1+\lambda+\lambda^{2}+\frac{8}{9} \lambda^{3}+\frac{46}{63} \lambda^{4}+\frac{2672}{4725} \lambda^{5} \\
& +\frac{183712}{439425} \lambda^{6}+\ldots
\end{aligned}
$$

This leads to $\langle w\rangle=1,\left\langle w^{2}\right\rangle=2,\left\langle w^{3}\right\rangle=16 / 3,\left\langle w^{4}\right\rangle=$ $368 / 21$, and so on. [Note that $\langle w\rangle=1$ is not determined by (7), but this is an immediate consequence of the initial condition (6).] One can also determine the fraction $S$ of individuals with no descendants (that is the probability that $w=0)$ by $S=g(-\infty)$. Clearly, $S=g(-\infty)$ is the solution of

$$
S=e^{2 S-2},
$$

and this gives $S=0.20318787 \ldots$

The power law $P(w) \sim w^{\beta}$ at small $w$ can also be easily understood from (7). If $P(w) \simeq A w^{\beta}$ for small $w$, one can write that as $\lambda \rightarrow-\infty$

$$
g(\lambda)-S \simeq A \Gamma(\beta+1)|\lambda|^{-\beta-1},
$$

and Eq. (7) gives [by the standard renormalization argument used to calculate exponents by linearization around a fixed point and which consists of writing the compensation of the singularities proportional to $|\lambda|^{-\beta-1}$ on both sides of (7)] that

$$
\beta=-\frac{\log S}{\log 2}-2 \simeq 0.2991138 \ldots,
$$

in excellent agreement with the results of the simulation. Other properties of the stationary distribution $P(w)$ could in principle be extracted from (7), but this would require more complicated mathematical developments. 
In this work, we have shown that a simple neutral model of sexual reproduction with nonoverlapping generations leads to a universal distribution of the weights of ancestors in genealogical trees. This universal distribution (more precisely its generating function) is the fixed point (7) of a simple renormalization Eq. (5). The exponent $\beta$ of the power law observed for small weights can be calculated exactly.

Our main result is that if we go very far in the past, about $80 \%$ of the (adult) population appears in the genealogical tree of every individual. If the weights of these ancestors represent how often they appear in this tree, these weights have a stationary probability distribution which is universal (i.e., independent of the generation and of the population size).

There are a number of extensions of the present work which, in our opinion, are worth pursuing. First, a more complete description of $P(w)$, in particular the large $w$ behavior, could be extracted from (7). If we wish to perform a better approximation to real genealogy, the possibility of overlaps between generations or of changes in the population size should be included. One can try to measure the distribution of lengths of segments in simple models $[7,11]$ for the evolution of chromosomes to see whether a power law in the length distribution is present there too. One could also investigate how our results would be modified by choosing instead of (4) a non-Poissonian distribution of offspring. Last, it would be interesting to consider the genealogical trees of several individuals to see how the repetitions on different trees are correlated [12].

With a little more imagination, one can construct other universality classes, by allowing the number $p$ of parents of each individual to be arbitrary, instead of $p=2$ in our earthly world. For general $p$, the fixed point Eq. (7) would become $g(\lambda)=\exp [-p+p g(\lambda / p)]$. There is no need to say that one might then try to expand the distribution $P(w)$, the fixed point $S$, or the exponent $\beta$ in powers of $\epsilon$ for $p=1+\epsilon$. In fact, one can show [12] that the case of an exponentially increasing (or decreasing) population size with $p=2$ parents for each individual is equivalent, as long as $g(\lambda)$ is concerned, to the case of a population of constant size with a number of parents $p$ which depends on the exponential growth rate of the population.

Apart from the potential application of our results to population genetics and evolutionary biology, the model of evolution studied here is connected to a number of problems of current interest in physics. First, the random assignment of the parents of individuals at each generation is very reminiscent of a problem of repartition of constraints introduced recently [13] to describe granular materials, with a recursion similar to (3). Graphs which locally look like trees but where large loops-responsible for cooperative effects - are present have attracted a lot of interest in the theory of disordered systems (spin glasses, localization) [14-19].

Last, the model studied here gives through (5) and (7) a very simple and pedagogical example of a problem with a nontrivial exponent, which can be solved exactly by a discrete renormalization transformation. One could try to see whether the oscillations [20] which usually accompany such discrete renormalization transformations are present here too.

Interesting discussions with Ugo Bastolla are gratefully acknowledged. S.C.M. acknowledges support from the Alexander von Humboldt Foundation (Germany) and from Fundación Antorchas (Argentina).

[1] G. Bräuer, Y. Yokoyama, C. Falguères, and E. Mbua, Nature (London) 386, 337 (1997).

[2] L. L. Cavalli-Sforza, Proc. Natl. Acad. Sci. U.S.A. 94, 7719 (1997).

[3] D. E. Reich and D. B. Goldstein, Proc. Natl. Acad. Sci. U.S.A. 95, 8119 (1998).

[4] S. Ohno, Proc. Natl. Acad. Sci. U.S.A. 93, 15276 (1996).

[5] Source: http://uts.cc.utexas.edu/ churchh/edw3chrt.html

[6] H. C. Harpending, M. A. Batzer, M. Gurven, L. B. Jorde, A. R. Rogers, and S.T. Sherry, Proc. Natl. Acad. Sci. U.S.A. 95, 1961 (1998).

[7] C. Wiuf and J. Hein, Genetics 147, 1459 (1997).

[8] C. Boehm, Am. Nat. 150, S100 (1997); D. S. Wilson, Science 276, 1816 (1997).

[9] S. A. Tishkoff, E. Dietzsch, W. Speed, A. J. Pakstis, J. R. Kidd, K. Cheung, B. Bonné-Tamir, A. S. SantachiaraBenerecetti, P. Moral, M. Krings, S. Pääbo, E. Watson, N. Risch, T. Jenkins, and K. K. Kidd, Science 271, 1380 (1996).

[10] M. Serva and L. Peliti, J. Phys. A 24, L705 (1991).

[11] B. Derrida and B. Jung-Muller, J. Stat. Phys. 94, 277 (1999).

[12] B. Derrida, S.C. Manrubia, and D.H. Zanette (to be published).

[13] S. N. Coppersmith, C.-h. Liu, S. N. Majumdar, O. Narayan, and T.A. Witten, Phys. Rev. E 53, 4673 (1996).

[14] H. Orland, J. Phys. (Paris), Lett. 46, L763 (1985).

[15] L. Viana and A. J. Bray, J. Phys. C 18, 3037 (1985).

[16] C. De Dominicis and P. Mottishaw, Europhys. Lett. 3, 87 (1987).

[17] I. Kanter and H. Sompolinsky, Phys. Rev. Lett. 58, 164 (1987).

[18] M. Mézard and G. Parisi, Europhys. Lett. 3, 1067 (1987).

[19] G. J. Rodgers and A.J. Bray, Phys. Rev. B 37, 3557 (1988).

[20] D. Sornette, Phys. Rep. 297, 239 (1998), and references therein. 